\title{
AUTOEFICACIA, AUTORREGULACIÓN Y MEDIDAS ANTROPOMÉTRICAS ASOCIADAS AL CONSUMO DE BEBIDAS ENDULZADAS EN JÓVENES DE MÉXICO
}

\section{SELF- EFFICACY, SELF- REGULATION AND ANTHROPOMETRIC MEASURES ASSOCIATED WITH CONSUMPTION OF SWEETENED BEVERAGES IN YOUTH OF MEXICO}

Jorge Palacios y Léa Hugo

Universidad del Valle de México, Querétaro. México

\section{Abstract}

One of the predisposing factors for developing overweight or obesity is the high consumption of sweetened beverages. Studies in this regard demonstrate an association between low levels of self-regulation and self-efficacy with weight management. Objective. To identify how cognitive factors and the consumption of sweetened beverages are related to nutritional status. Method. Anthropometric measurements were made and the frequency of consumption of sweetened beverages was indicated. In addition, the self-efficacy scale of eating behavior and the self-regulation scale of sweetened beverages were applied in a sample of 113 students from Querétaro, Mexico. Results. The frequency of soda consumption was $38.9 \%$ for the cola flavor. Participants have a BMI of $21.07 \mathrm{~kg} / \mathrm{m}^{2}$ ( \pm 8.7 ), equivalent to normal weight. Additionally, the frequency of consumption of sweetened beverages is positively related to the increase in visceral fat and the waist-hip indicator. Self-efficacy and self-regulation are negatively related to the consumption of sweetened beverages. Conclusions. The consumption of sweetened beverages generates an increase in weight and cognitive factors allow regulating the consumption of these beverages, so they can be considered as health care promoters.

Keywords: Consumption of soda, self-efficacy, self-regulation, anthropometry, youth.

\section{Resumen}

Uno de los factores predisponentes para desarrollar sobrepeso u obesidad, es el alto consumo de bebidas endulzadas. Los estudios al respecto demuestran una asociación entre bajos niveles de autorregulación y autoeficacia con el manejo del peso. Objetivo. Identificar de qué manera se relacionan los factores cognitivos y el consumo de bebidas endulzadas con el estado de nutrición. Método. Se realizaron mediciones antropométricas y se registró la frecuencia de consumo de bebidas endulzadas. Además, se aplicaron la escala de autoeficacia de la conducta alimentaria y la escala de autorregulación de bebidas endulzadas en una muestra de 113 estudiantes de Querétaro, México. Resultados. La frecuencia del consumo de refresco fue de $38.9 \%$ para el sabor cola. Los participantes tienen un IMC de 21.07 $\mathrm{kg} / \mathrm{m}^{2}( \pm 8.7)$, equivalente a normopeso. Adicionalmente la frecuencia del consumo de bebidas endulzadas se relaciona positivamente con el aumento de la grasa visceral y el indicador de cintura-cadera. Autoeficacia y autorregulación se relacionan negativamente con el consumo de bebidas endulzadas. Conclusiones. El consumo de bebidas endulzadas genera un aumento de peso y los factores cognoscitivos permiten regular el consumo de estas bebidas, por lo que pueden ser considerados promotores del cuidado de la salud.

Palabras clave: Consumo de refresco, autoeficacia, autorregulación, antropometría, jóvenes. 
La Organización Mundial de la Salud (OMS, 2018), define el sobrepeso y obesidad como una acumulación anormal o excesiva de grasa perjudicial para la salud. Las tasas de sobrepeso y obesidad han aumentado a nivel nacional y mundial, sus causas son numerosas y complejas involucrando componentes genéticos, psicológicos, sociales y ambientales.

Según la Encuesta Nacional de Salud y Nutrición (ENSANUT) en 2016 se reportó que la prevalencia del sobrepeso y la obesidad combinada en México es de 72.5\%. Las personas adultas de 18 o más años tienen $39.2 \%$ de sobrepeso y $33.3 \%$ son obesos. Además 4 de cada 10 adolescentes de 12 a 19 años de edad presentan sobrepeso u obesidad, con una prevalencia de 36.3\%, mientras que en adultos mayores de 20 años en adelante, 7 cada de 10 adultos padecen un exceso de peso. Una de las causas del sobrepeso y la obesidad es el desequilibrio energético entre calorías consumidas y gastadas, debido a un aumento en la ingesta de alimentos de alto contenido calórico o un descenso en la actividad física (Rodríguez, Avalos \& López, 2014), impactando sobre la expectativa y calidad de vida de una persona (Malik, Popkin, Bray, Dosesprés, Willett \& Hu, 2010).

Uno de los factores predisponentes para desarrollar sobrepeso $\mathrm{u}$ obesidad es el alto consumo de bebidas endulzadas (López, 2008), debido a sus características de refinamiento, alta densidad energética, alto aporte de grasas saturadas y colesterol, además de un bajo consumo de fibra dietética (García, López, Moreno, Miranda \& Zepeda, 2018). Las bebidas endulzadas se componen de refrescos, bebidas de frutas, zumos y bebidas energéticas que han aumentado en diversas presentaciones y marcas en todo el mundo. Específicamente las bebidas endulzadas conducen al aumento de peso en virtud de su alto contenido de azúcar y la compensación incompleta de la energía total en las comidas posteriores de la ingestión de calorías líquidas. Debido al alto contenido de carbohidratos, rápidamente absorbibles como sacarosa (50\% de glucosa y $50 \%$ de fructosa) y jarabe de maíz de alta fructosa (con mayor frecuencia $45 \%$ de glucosa y 55\% de fructosa), junto con los volúmenes consumidos, las bebidas azucaradas pueden aumentar el riesgo de síndrome metabólico y diabetes tipo 2 , no solo a través de la obesidad sino también al aumentar la carga glucémica en la dieta, lo que lleva a la resistencia a la insulina, la disfunción celular y la inflamación (Fagherazzi, Vilier, Saes
Sartorelli, Lajous, Balkau \& Clavel-Chapelon, 2013). Se ha encontrado una relación directa en niños con obesidad por consumir bebidas endulzadas, ya que tienen un consumo de energía más alta, siguiendo ese patrón hasta la vida adulta, además de tener efectos que pueden conducir al desarrollo de enfermedades crónicas degenerativas como hipertensión arterial y/o diabetes, promoviendo la acumulación de tejido adiposo visceral y elevando la lipogénesis hepática lo que ayuda a padecer de triglicéridos y colesterol LDL altos, además de colesterol HDL bajos (Malik, Popkin, Bray, Després, Willett \& $\mathrm{Hu}, 2010)$.

Existe evidencia que menciona que las bebidas endulzadas tienen poca capacidad de saciedad e implican una pobre compensación dietética. Un estudio sobre las sensaciones del apetito (hambre, saciedad e ingestión posterior a una comida) apoya la idea de que los líquidos tienen menor capacidad de producir saciedad que los alimentos sólidos (García, 2018). Por estas razones, es de esperar que la ingestión de bebidas con aporte energético se vincule con un equilibrio positivo de energía y obesidad (Rodríguez, Avalos \& López, 2014). El efecto de las bebidas endulzadas con el sobrepeso y la obesidad depende principalmente por la distribución actual del consumo de estas bebidas y la magnitud del efecto de estas en el índice de masa corporal (IMC) u otras medidas del estado del peso (Forshee, Anderson \& Storey, 2008).

Adicionalmente, en algunas de las bebidas endulzadas se agregan edulcorantes no nutritivos (ENN), lo cuales se han utilizado como un sustituto del azúcar para darle menos calorías a la bebida y más sabor dulce y en las bebidas tipo soda sin calorías (light o zero) contienen más aporte de ENN de la ingesta alimentaria, asociándolos como una medida nutricional para reducir la ingesta de azúcar y calorías o controlar su peso (Durán, Record, Encina, Salazar de Ariza, Cordón, Cereceda, et al, 2015). Un estudio previo ha encontrado que la sucralosa (un edulcorante presente en la mayoría de las bebidas azucaradas), estimula el circuito de recompensa, como lo realiza la sacarosa, pero con menor magnitud, sin satisfacer plenamente el deseo de la ingesta calórica del sabor dulce, lo que puede ayudar al incremento excesivo de la ingesta alimentaria (Frank, Oberndorfer, Simmons, Paulus, Fudge, Yang \& Kaye, 2008). Fowler, Williams, Resendez, Hunt, Hazuda \& Stern, (2008) encontraron que los edulcorantes no nutritivos pueden incrementar el apetito post ingesta de la bebida endulzada, mientras que 
la ingesta de tres o más de estas bebidas por día promueve el aumento de peso dos veces más que si no se consumiera (Mattes \& Popkin, 2008).

Por otra parte, existen estudios que demuestran asociación entre bajos niveles de autorregulación y la autoeficacia con el manejo del peso (Román, Díaz, Cárdenas \& Lugli, 2007), el control alimenticio (Dutton, Davis, Rhode \& Brantley, 2004), la alimentación saludable (Wilson-Barlow, Hollins \& Clopton, 2014), la conducta alimentaria (Ruiz, Berrocal, López \& Rivas, 2003), así como la autorregulación de la alimentación (Ames, Wurpts, Pike, MacKinnon, Reynolds \& Stacy, 2016; Hughes, Power \& O'Connor, 2015; Johnson, Pratt \& Wardle, 2012). En Latinoamérica, Lugli, Arzolar \& Vivas (2009), analizaron las habilidades relacionadas con el control del peso corporal y encontraron que la autorregulación en hábitos alimentarios alude a las destrezas cognitivas y conductuales requeridas por las personas que desean bajar de peso e implica un esfuerzo constante y progresivo para controlar la ingesta alimentaria y cumplir con una rutina de ejercicios de forma regular.

En México, se han realizado algunos estudios para medir la regulación asociada al constructo de autoeficacia (Flores, González-Celis, \& Valencia, 2010; Guzmán \& Gómez, 2011; Palacios, Ramírez, Anaya, Hernández \& Martínez, 2017). En cuanto a la autorregulación, Campos, Argüelles \& Lugli, (2012), encontraron que la presencia de obesidad se asoció con bajos niveles de autorregulación de hábitos alimentarios. Por su parte, Uscanga, González, Del Moral \& Hernández (2017), mostraban que una menor autorregulación se asocia con el aumento del IMC, confirmando que no solo es útil en el tratamiento sino también en la prevención de la obesidad. Recientemente, Palacios (2019), señalaba las dificultades que los jóvenes tienen para regular su afecto para consumir bebidas endulzadas, además de tener un decremento en su control cognitivo frente a las bebidas endulzadas, ambos (regulación afectiva y cognitiva) contribuyen aumentando el nivel de consumo de refresco. Concluía proponiendo la utilidad de evaluar la autorregulación del consumo de bebidas endulzadas como un precursor de los problemas del control de peso.

Por otra parte, los estudiantes universitarios podrían mantener un estilo de vida que favoreciese la ingestión inadecuada de alimentos, ocasionando cambios en su peso corporal. La alimentación de estos jóvenes generalmente se ve modificada como resultado de los cambios de tipo social, económico y cultural que se presentan en su etapa de desarrollo y formación profesional, lo que les ocasiona una mala nutrición, tanto por deficiencia de nutrimentos como por exceso de calorías; por ello, su dieta se caracteriza por consumo de grandes cantidades de carnes con grasa saturada, refrescos, postres y dulces, teniendo una ingesta inadecuada de verduras y frutas con un ambiente de ejercicio o actividad física escasa o nula (Franco \& Miramontes, 2013). Debido al estilo de vida de los universitarios, se considera relevante estudiar los factores psicológicos presentes en el consumo frecuente de bebidas endulzadas, identificando que los factores cognitivos son precursores del comportamiento (Bandura, 2001; Palacios, 2019a; Palacios \& Bustos, 2012; Palacios \& Soler, 2017). Estos factores se han utilizado para comprender diferentes conductas de riesgo para salud ya que tienen capacidad descriptiva y explicativa de los aspectos generales del comportamiento (Bandura, 1977, 1998; Palacios, 2015).

A partir de la evidencia encontrada respecto a los factores cognitivos involucrados en el consumo de bebidas, se busca corroborar la relación entre el consumo de bebidas endulzadas con parámetros de antropometría para indagar su posible asociación con la composición corporal de un individuo en un ambiente universitario, ya que se ha visto en varios estudios la asociación del aumento de peso con este tipo de bebidas (Malik et al., 2010; López, 2008). Considerando estos determinantes, el objetivo de la presente investigación fue conocer de qué manera se relacionaban los factores cognitivos (autoeficacia y autorregulación) y el consumo de bebidas endulzadas, con el estado de nutrición (medidas antropométricas) de una muestra de jóvenes estudiantes del estado de Querétaro, México.

Se propuso como hipótesis que la autoeficacia, la autorregulación y las medidas antropométricas tendrían una relación con el consumo de bebidas endulzadas de los jóvenes. 


\section{MÉTODO}

\section{Diseño y participantes}

Para la presente investigación se realizó un estudio de campo con un diseño no experimental de corte transversal y de tipo correlacional (Montero \& León, 2002). Se seleccionó una muestra no probabilística de tipo intencional de 113 estudiantes, 56 mujeres (49.6\%) y 57 hombres (50.4\%) con un promedio de edad de 20.9 años $(D T=1.7)$ de una universidad privada del estado de Querétaro, México. Se consideraron como criterios de inclusión que los participantes tuvieran entre 18 y 24 años de edad, que fueran estudiantes de alguna licenciatura o ingeniería de la universidad, no presentaran alguna enfermedad crónica degenerativa. Como criterios de exclusión, se consideraron no haber realizado esfuerzo físico previo a la medición antropométrica, haber ingerido alcohol durante la última semana, estar en etapa de embarazo, presentar amputación de extremidades del cuerpo y presentar atrofia muscular o deformación del sistema esquelético.

\section{Instrumentos}

Bebidas endulzadas. La medición del consumo de bebidas endulzadas se realizó por medio de siete ítems elaborados ad hoc para este estudio, agrupados en una escala tipo Likert sobre la frecuencia de consumo de refresco por semana, con niveles de respuesta que van de un consumo poco frecuente (1 o 2 días/semana), medianamente frecuente (3 a 6 días/semana) hasta consumo diario (7 días/semana) y el tipo de refresco ingerido (sabor -manzana, toronja, naranja, limón, uva-, cola o light). Para el presente estudio se obtuvo un nivel alfa de Cronbach de .75 (IC95\%=.66 -.81), para las bebidas utilizadas. La escala tiene validez de contenido y de criterio con el consumo de refresco como se ha demostrado en estudios previos (Campos-Ramírez, Palacios, Anaya-Loyola \& Ramírez-Amaya, 2019).

Indicadores antropométricos. El sobrepeso y la obesidad se midieron por medio del índice de masa corporal (IMC), considerando a una persona con un IMC igual o superior a $30 \mathrm{~kg} / \mathrm{m}^{2}$ como obesa y con un IMC igual o superior a $25 \mathrm{~kg} / \mathrm{m}^{2}$ es considerada con sobrepeso. Para complementar la evaluación de la adiposidad, se utilizó la medida de Circunferencia de Cintura (CC) para predecir un posible factor de riesgo asociado a enfermedades y/o eventos cardiovasculares, además de desórdenes metabólicos como diabetes o síndrome metabólico (Briones, Calacuayo, Olivares, Gaytán, González, Luévano, Ríos \& Palos, 2018). Para las mediciones antropométricas se utilizó un estadímetro marca SECA®-5700211115667, una báscula electrónica para impedancia bioeléctrica marca TANITA®-331S con electrodos en manos y pies, que permitío obtener la información de peso, grasa, masa muscular y edad metabólica, así como una cinta métrica SECA®-201.

Factores cognitivos. La evaluación de la autoeficacia alimentaria se realizó con la versión modificada del instrumento de Palacios, Ramírez, Anaya, Hernández \& Martínez, (2017) para el consumo de refresco y alimentos. Para el presente estudio se integraron nueve ítems sobre el consumo de bebidas endulzadas. La medición final se conforma por 12 ítems de estimación numérica, con opción de respuesta que va de 1 a 10, que permite estimar la capacidad de evitar consumir bebidas endulzadas (p. e., Puedo dejar de tomar refresco y beber agua natural). La medida se integra por dos factores referentes a (1) La capacidad de moderación del consumo de refresco $(\alpha=.79)$ y (2) La capacidad de cambiar bebidas endulzadas por bebidas naturales $(\alpha=.80)$. La escala utilizada posee validez de constructo, de criterio con el IMC de las personas y predictiva con los problemas de riesgo alimentario (Palacios \& Ramírez, 2016), así como niveles de confiabilidad alfa de Cronbach de .80 (IC95\% = .76- .83) para el total del instrumento.

Para evaluar la autorregulación que tiene una persona para controlar su consumo de bebidas endulzadas, se utilizó el instrumento de Palacios (2019b). El instrumento se conforma por 15 ítems tipo Likert, con opción de respuesta de nunca a siempre. La escala se divide en dos factores (1) regulación cognitiva y (2) regulación afectiva del consumo de bebidas endulzadas. Cuenta con un índice de consistencia interna de .81 (IC95\% = .78- .84) para el factor de regulación cognitiva y un índice de .87 (IC95\% = .85- .89) para el factor de regulación afectiva. La escala cuenta con validez de constructo, factorial, de criterio y predictiva del consumo de refresco en jóvenes de México.

\section{Procedimiento}

Se aplicó el instrumento de forma individual a los participantes dentro de las instalaciones de la universidad. El tiempo promedio para las mediciones antropométricas y la respuesta a la escala fue de 20 a 30 
minutos. Previo a la presentación del instrumento se les explicó que se trataba de un cuestionario para conocer algunas actividades relacionadas con su alimentación. Para la toma de mediciones antropométricas, se utilizó el estadímetro para tomar la talla, donde el individuo tiene que estar parado y de espaldas; también se determinaron las medidas antropométricas [peso, porcentaje de grasa corporal (\%G), Índice de Masa Corporal (IMC), Índice de cintura-cadera (ICC), agua en kilogramos y la masa muscular también en kilogramos], para ello los participantes fueron evaluados sin calzado, en pantaloneta y camiseta ubicándose en el centro de la balanza (Cardozo, Guzman \& Torres, 2016). Para la medición de la circunferencia de cintura el individuo tuvo los brazos relajados a los costados en posición anatómica y el abdomen descubierto, con la cinta métrica se medió la circunferencia colocándola alrededor de la cintura para tomar la lectura correspondiente. Mientras que, para la medición de la circunferencia de cadera, se palpó los trocánteres del fémur y se colocó la cinta métrica sin comprimirla alrededor de estos, en su circunferencia máxima y se procedió a realizar su lectura.

\section{Ánalisis de datos}

Los análisis de datos se realizaron considerando la estadística descriptiva para las bebidas endulzadas y para los indicadores antropométricos se obtuvieron medidas de tendencia central y dispersión. Se utilizó la prueba de correlación Rho de Spearman para asociar el consumo de los diferentes sabores de las bebidas endulzadas con los indicadores antropométricos, así como para las asociaciones entre las bebidas endulzadas y los factores cognoscitivos, mediante el programa SPSS 14.

\section{Consideraciones éticas}

A todos los participantes se les aclaró que la información era anónima, se les garantizó la confidencialidad de los datos proporcionados y se resolvieron las dudas que tuvieran con respecto a su participación. En primer lugar, se solicitó el permiso para realizar el estudio a las autoridades correspondientes, una vez concedido se llevó a cabo la investigación. El estudio fue revisado y aprobado por el Comité de Bioética y de Investigación de la Universidad del Valle de México, campus Querétaro. A cada participante se le pidió su colaboración mediante la firma de una carta de consentimiento informado. El protocolo de la presente investigación fue establecido de acuerdo al Reglamento de la Ley General de Salud, considerando el apartado sobre investigación en seres humanos (Secretaria de Salud, 2011).

\section{RESULTADOS}

La frecuencia del consumo mostró que la mayoría de los estudiantes encuestados reporta no consumir refresco, sin embargo, existe una proporción de estudiantes que lo consume con frecuencia. La Tabla 1 muestra los resultados de la frecuencia de consumo en donde destacan como refrescos más consumidos el de sabor manzana y de cola.

Tabla 1. Frecuencia de consumo de bebidas endulzadas con diferentes sabores

\begin{tabular}{lccccc}
\hline & Ninguna & $\begin{array}{c}\mathbf{1} \text { o } \mathbf{2} \\
\text { veces }\end{array}$ & $\begin{array}{c}\mathbf{3 - 4} \\
\text { veces }\end{array}$ & $\begin{array}{c}\mathbf{5 - 6} \\
\text { veces }\end{array}$ & 7 veces \\
\hline Manzana & 72.6 & 23.9 & 2.7 & --- & 9.0 \\
Naranja & 84.1 & 14.2 & 1.8 & --- & --- \\
Limón & 85.0 & 14.2 & 0.9 & --- & --- \\
Uva & 88.5 & 9.7 & 0.9 & 0.9 & --- \\
Toronja & 87.6 & 9.7 & 1.8 & --- & 0.9 \\
Cola & 43.4 & 38.9 & 10.6 & 2.7 & 4.4 \\
Light & 89.4 & 7.1 & 1.8 & 0.9 & 0.9 \\
\hline
\end{tabular}

Nota: valores porcentuales; $N=113$.

Respecto a los valores obtenidos de las mediciones antropométricas (Tabla 2), se observa que el IMC es de $21.07 \mathrm{~kg} / \mathrm{m}^{2}$ ( \pm 8.7 ), lo que indica que los estudiantes se encuentran en normopeso, sin embargo, el valor máximo se ubica en obesidad grado I. El porcentaje de grasa está en el rango de saludable (20-30\%) tomando en cuenta a los hombres y mujeres del estudio. El ICC arroja un riesgo poco elevado $(0.87 \pm 1.1)$; además, se puede observar que la masa muscular está en un promedio de 40.48 kg ( \pm 18.45).

Se realizaron correlaciones con la prueba Rho de Spearman entre los diferentes tipos de bebidas endulzadas y los indicadores antropométricos. La Tabla 3 muestra que algunos indicadores antropométricos correlacionan de forma positiva y significativa con las bebidas endulzadas. El consumo de refresco de sabor manzana se relaciona positivamente con el ICC; las bebidas endulzadas de sabor naranja con la circunferencia de cintura, mientras que el refresco de limón con la grasa visceral. La bebida endulzada de toronja se correlaciona con la grasa visceral y con el ICC 
de forma positiva. Las bebidas tipo cola tienen correlación positiva con la masa muscular, circunferencia de cintura y con el ICC. El consumo de refresco endulzado tipo light se relaciona positiva y significativamente con la grasa visceral, el IMC, la circunferencia de cadera y la grasa corporal.

Tabla 2. Descripción de valores antropométricos

\begin{tabular}{|c|c|c|c|c|}
\hline & $M$ & $D T$ & Mín. & Máx. \\
\hline Peso (kg) & 59.3 & 23.8 & 53.0 & 108.6 \\
\hline Talla (cm) & 166.1 & 8.4 & 147.0 & 187.0 \\
\hline IMC $\left(\mathrm{kg} / \mathrm{m}^{2}\right)$ & 21.1 & 8.7 & 19.0 & 34.7 \\
\hline ICC & 0.9 & 1.1 & 0.7 & 1.8 \\
\hline $\begin{array}{l}\text { Porcentaje de } \\
\text { grasa (\%) }\end{array}$ & 20.5 & 9.8 & 13.0 & 40.2 \\
\hline Grasa visceral & 3.3 & 2.8 & 1.0 & 12.0 \\
\hline $\begin{array}{l}\text { Masa muscular } \\
(\mathrm{kg})\end{array}$ & 40.5 & 18.5 & 3.7 & 68.9 \\
\hline $\begin{array}{l}\text { Edad Metabólica } \\
\text { (años) }\end{array}$ & 26.3 & 3.2 & 12.0 & 33.5 \\
\hline Cintura $(\mathrm{cm})$ & 42.2 & 38.4 & 63.0 & 107.5 \\
\hline Cadera $(\mathrm{cm})$ & 94.8 & 9.2 & 42.0 & 114.0 \\
\hline
\end{tabular}

Tabla 3. Correlaciones entre las diferentes bebidas endulzadas y los indicadores antropométricos

\begin{tabular}{cccccccccc}
\hline & $\mathbf{P}$ & $\mathbf{G}$ & $\mathbf{M M}$ & $\mathbf{G V}$ & IMC & $\mathbf{C}$ & $\mathbf{C A}$ & ICC & GC \\
\hline $\mathbf{M}$ & .09 & -.07 & .01 & .18 & .09 & .13 & .15 & $.28^{* *}$ & .09 \\
$\mathbf{N}$ & .01 & -.03 & .06 & .15 & -.02 & $.26^{* *}$ & .09 & .16 & .10 \\
$\mathbf{L}$ & .11 & -.01 & .14 & $.21^{*}$ & .10 & .13 & .09 & .12 & .06 \\
$\mathbf{U}$ & .05 & -.15 & .08 & .09 & -.03 & .14 & -.00 & .18 & -.02 \\
$\mathbf{T}$ & .17 & -.08 & .14 & $.21^{*}$ & .09 & .06 & .08 & $.30^{* *}$ & .07 \\
$\mathbf{C}$ & .17 & .04 & $.19^{*}$ & .16 & .16 & $.27^{* *}$ & .08 & $.28^{* *}$ & .04 \\
$\mathbf{L}$ & .10 & .12 & -.04 & $.19^{*}$ & $.25^{* *}$ & .15 & $.20^{*}$ & .09 & $.19^{*}$ \\
\hline
\end{tabular}

Nota: M=Manzana; N=Naranja; L=Limón; U=Uva; T=Toronja; C=Cola; L=Light; $\mathrm{P}=$ Peso; $\mathrm{G}=$ Grasa; $\mathrm{MM}=$ Masa Muscular; $\mathrm{GV}=$ Grasa Visceral; IMC=Índice de Masa Corporal; $C=C$ intura; $C A=C$ adera; ICC=Índice CinturaCadera; GC=Grasa Corporal; ${ }^{*} p<.05 * * p<.01 * * * p<.00$.

En las correlaciones entre el consumo de las diferentes bebidas y los factores cognitivos, se observa que el consumo de refresco en sus diferentes sabores presenta una correlación negativa y estadísticamente significativa con la autoeficacia, siendo la magnitud más alta con la bebida sabor uva. El control cognitivo se asocia negativa y significativamente con todos los sabores de las bebidas endulzadas, excepto con el consumo de bebidas light. Adicionalmente la reguación afectiva se encuentra positiva y significativamente relacionada con el consumo de bebidas endulzadas sabor limón, uva, toronja y cola (Tabla 4).
Tabla 4. Correlaciones entre las diferentes bebidas endulzadas con los factores cognitivos

\begin{tabular}{lccc}
\hline & Autoeficacia & $\begin{array}{c}\text { Autorregulación } \\
\text { cognitiva }\end{array}$ & $\begin{array}{c}\text { Autorregulación } \\
\text { afectiva }\end{array}$ \\
\hline Manzana & $-.29^{* *}$ & $-.32^{* *}$ & .18 \\
Naranja & $-.29^{* *}$ & $-.21^{*}$ & .09 \\
Limón & $-.32^{* *}$ & $-.24^{*}$ & $.24^{*}$ \\
Uva & $-.36^{* *}$ & $-.30^{* *}$ & $.27^{* *}$ \\
Toronja & $-.24^{*}$ & $-.25^{* *}$ & $.19^{*}$ \\
Cola & $-.28^{* *}$ & $-.32^{* *}$ & $.31^{* *}$ \\
Light & -.18 & -.07 & .04 \\
\hline Nota: ${ }^{*} p<.05 * * p<.01 * * * p<.001$ & & \\
& & & \\
DISCUSIÓN & & & \\
\hline
\end{tabular}

En el presente estudio se determinó la relación entre el consumo de bebidas endulzadas, las medidas antropométricas y los factores cognitivos de autoeficacia y autorregulación de bebidas en un grupo de estudiantes universitarios. Los resultados obtenidos muestran valores promedio de normopeso debido a que los participantes tienen un IMC de $21.07 \mathrm{~kg} / \mathrm{m}^{2}$, un porcentaje de grasa de $20.5 \%$ y un promedio de $40.5 \mathrm{~kg}$ de músculo en la mayoría de los estudiantes tanto hombres como mujeres. Comparado con las cifras de sobrepeso u obesidad de la ENSANUT (2016), la mayoría de los estudiantes presentan un rango de peso saludable, aunque se puede observar un IMC mínimo de 19.0 y un máximo de $34.7 \mathrm{~kg} / \mathrm{m}^{2}$ obteniendo un índice de sobrepeso y obesidad en esta población estudiantil.

También encontramos que la mayoría de los estudiantes universitarios no consumen refresco en la semana, pero algunos consumen de 1 a 2 veces por semana bebidas endulzadas. Al respecto, algunos estudios mencionan que los edulcorantes tienen la capacidad de darle menos calorías y más sabor a las bebidas, pero por efecto del mismo pueden ayudar a que exista un exceso en la ingesta alimentaria incidiendo con el aumento de peso o de enfermedades metabólicas (Duran, 2015; Fowler, 2008; Frank, 2008; Mattes, 2008).

Respecto al consumo de refresco, podemos mencionar que la asociación con la adiposidad parece ser más pronunciada cuando hay un incremento en el consumo de bebidas endulzadas en la población joven universitaria de nuestro estudio. Existe una interacción significativa entre un factor dietético: -ingesta de bebidas endulzadas- y un marcador de predisposición genética, 
obesidad, y el riesgo de obesidad (López, 2008; OMS, 2018). Los resultados corroboran que los jóvenes con sobrepeso y obesidad de acuerdo con su porcentaje de grasa e índice de masa corporal respectivamente tienen un mayor consumo de bebidas endulzadas sabor cola. Una alimentación saludable, no requiere de bebidas que aporten energía a la dieta (bebidas endulzadas, bebidas de cola, mucho menos jugos industrializados o naturales), el agua o las bebidas no calóricas, son capaces de satisfacer las necesidades hídricas del individuo, además de ayudar a frenar la ganancia de peso en individuos con sobrepeso y obesidad (García, 2018).

Por otra parte, al analizar la relación entre las bebidas carbonatadas y/o endulzadas con las medidas antropométricas (grasa corporal total, IMC, porcentaje de grasa, grasa visceral, grasa corporal, índice de circunferencia de cintura - cadera); se observa que los participantes que consumen refrescos de tipo light o de sabores a fruta presentan valores más elevados de grasa corporal total e ICC. Los resultados coinciden con estudios previos que señalan una correlación entre el consumo de refrescos y el aumento de peso en este tipo de población (Malik et al., 2010; Rivera, 2008). Aunado a lo anterior, el cambio socio-psicológico de la población estudiantil también se ve reflejado en el estilo de vida y la alimentación de los estudiantes (Franco, 2013), promoviendo una acumulación anormal de grasa por el consumo de bebidas endulzadas debido a las calorías extras que aporta este tipo de bebidas, por lo general en el abdomen (Rodríguez et al., 2014), contribuyendo a desarrollar enfermedades cardiovasculares, diabetes y síndrome metabólico en adultos jóvenes (Malik et al., 2010). Un consumo excesivo de bebidas endulzadas no solo afecta antropométrica y metabólicamente al individuo, ya que si se tiene una baja regulación cognitiva, autoeficacia y dificultades de regulación afectiva se puede producir un abandono en la buena salud personal y nutricional.

La evidencia encontrada respecto a la asociación positiva entre los factores cognitivos y el consumo de refrescos indica la relación de estos factores como predictores del consumo de bebidas endulzadas. Los hallazgos aportan una fuerte evidencia a la teoría social cognitiva propuesta por Bandura $(1977,1998,2001)$ que explica la conducta centrándose sobre factores ambientales, afectivos y cognitivos. De acuerdo con esta teoría (Bandura, 1997, 2001), hay dos mecanismos involucrados en la adquisición y regulación de la conducta. El primero hace referencia a la experiencia directa recibida en cada una de nuestras acciones (autoeficacia) y que son la base del aprendizaje vicario y el modelado. El segundo mecanismo tiene que ver con la regulación del comportamiento, ambos constructos son considerados como los mejores predictores de las acciones (Palacios \& Bustos, 2012; Palacios, 2019a). La capacidad de eficacia, así como la regulación cognitiva y afectiva se ha puesto a prueba en la presente investigación para determinar el grado en el cual los individuos consumen bebidas endulzadas. Se ha encontrado que un alto grado de autoeficacia y de regulación cognitiva, así como una adecuada regulación afectiva, correlaciona con un bajo consumo de bebidas endulzadas en sus diferentes sabores, lo cual puede considerarse un comportamiento saludable (Palacios et al., 2017; Palacios, 2019b). Los datos aquí encontrados corroboran lo reportado en investigaciones previas (Ames et al., 2016; Campos, Argüelles \& Lugli, 2012; Guzmán \& Gómez, 2011; Lugli et al., 2009; Palacios, 2019b; Palacios et al., 2017; Román et al., 2007; WilsonBarlow et al., 2014), donde se ha establecido una relación entre factores cognitivos y conductas alimentarias, de manera que las variables cognitivas (autoeficacia y autorregulación) incorporadas en este estudio pueden resultar relevantes para la adquisición, mantenimiento, moldeamiento y deshabituación del consumo de bebidas endulzadas.

\section{Conclusiones}

La detección precoz y el tratamiento del sobrepeso y la obesidad deberían ser objetivos prioritarios para los servicios de salud. El abordaje y el tratamiento de estas problemáticas se realiza habitualmente por los nutricionistas que pueden realizar modificaciones en la alimentación desde un cambio en los horarios de comida hasta la reducción o eliminación de ciertos alimentos como los alimentos con alto contenido de grasa o azúcar así como la promover la actividad física. El nivel de asociación entre los factores cognitivos (autoeficacia y autorregulación) con el consumo de bebidas endulzadas en sus diferentes sabores y la implicación de las bebidas endulzadas en el estado de nutrición de los jóvenes configuran las principales aportaciones de este estudio. Conforme a estos resultados, la efectividad de las intervenciones de salud nutricional sería mayor si se complementaran con el apoyo de profesionionales de la 
psicología que realicen intervenciones cognitivoconductuales o sociocognitivas (Bandura, 1998, 2001; Palacios \& Bustos, 2012) dirigidas a modificar los mecanismos de autorregulación y autoeficacia de las personas que buscan regular su peso.

Es importante disminuir el consumo de bebidas endulzadas en México, ya que como se ha mostrado en los resultados, las calorías extras aportadas por estas bebidas contribuyen a un posible aumento de peso como consecuencia del exceso de azúcar que se convierte en grasa (Rodríguez et al., 2014), aumentando el riesgo de desarrollar alguna enfermedad crónico degenerativa o cardiovascular (Malik et al., 2010). Los edulcorantes no nutritivos (ENN) presentes en estas bebidas ayudan a aumentar la grasa corporal o el apetito, generando un exceso de energía en la dieta del individuo y favoreciendo el aumento de peso y grasa en el cuerpo (Duran, 2015; Fowler, 2008; Frank, 2008; Mattes, 2008).

\section{Limitaciones}

La principal limitación se relaciona con el tamaño de la muestra que no resulta representativa de la población universitaria, además de no haberse realizado de forma probabilística, por lo que los resultados de este estudio no se pueden generalizar a toda la población universitaria. Además, el tipo de análisis efectuado no permite realizar afirmaciones de causalidad.

Para dar continuidad a los resultados de esta investigación, se pretenden incorporar factores afectivos y neuroconductuales involucrados en el consumo de bebidas endulzadas en futuros estudios.

\section{REFERENCIAS}

Ames, S., Wurpts, I., Pike, J., MacKinnon, D., Reynolds, K. \& Stacy, A. (2016). Self-regulation interventions to reduce consumption of sugarsweetened beverages in adolescents. Appetite, 105, 652-662. doi:10.1016/j.appet.2016.06.036

Bandura, A. (1977). Self-efficacy: Toward a unifying theory of behavioral change. Psychological Review, 84(2), 191- 215. doi:10.1016/01466402(78)90002-4

Bandura, A. (1998). Health promotion from the perspective of social cognitive theory. Psychology and Health, 13, 623-649. doi:10.1080/08870449808407422

Bandura, A. (2001). Social cognitive theory: An agentic perspective. Annual Review of Psychology, 52, 1-26. doi:10.1146/annurev.psych.52.1.1
Briones, Z., Calacuayo, L., Olivares, J., Gaytán, D., González, O., Luévano, C. Ríos, M. \& Palos A. (2018). Índice de masa corporal y circunferencia de cintura: ¿se relacionan con el consumo de bebidas azucaradas en una población adulta mexicana? Revista Mexicana de Endocrinología, Metabolismo y Nutrición, 5, 53- 59.

Campos, Y., Argüelles, V., \& Lugli, Z. (2012). Autorregulación y autoeficacia en estudiantes universitarios con obesidad. UniverSalud, 8(16), 3-8.

Campos-Ramírez, C., Palacios, J., Anaya-Loyola, A., \& Ramírez-Amaya, V. (2019). Evaluación de los factores de la teoría de la conducta planeada en relación con el patrón de consumo de bebidas carbonatadas en jóvenes universitarios. Revista Chilena de Nutrición, 46(3), 319- 327. doi:10.4067/S0717-75182019000300319

Cardozo, L., Guzman, Y., \& Torres, J. (2016). Porcentaje de grasa corporal y prevalencia de sobrepeso-obesidad en estudiantes universitarios de rendimiento deportivo de Bogotá, Colombia. Nutrición Clínica y Dietética Hospitalaria, 36(3), 68-75. doi:10.12873/363cardozo

Durán, S., Record, J., Encina, C., Salazar de Ariza, J., Cordón, K., Cereceda, M., Antezana, S., \& Espinoza, S. (2015). Consumo de edulcorantes no nutritivos en bebidas carbonatadas en estudiantes universitarios de algunos países de Latinoamérica. Nutrición Hospitalaria, 31(2), 959-965. doi:10.3305/nh.2015.31.2.8026

Dutton G., Davis M., Rhode P., \& Brantley P. (2004). Use of the Weight Efficacy Lifestyle Questionnaire with African American women: Validation and extension of previous findings. Eating Behavior, 5, 375-384. doi:10.1016/j.eatbeh.2004.04.005

Fagherazzi, G., Vilier, A., Saes Sartorelli, D., Lajous, M., Balkau, B., \& ClavelChapelon, F. (2013). Consumption of artificially and sugarsweetened beverages and incident type 2 diabetes in the Etude Epidémiologique auprès des femmes de la Mutuelle Générale de I'Education Nationale-European Prospective Investigation into Cancer and Nutrition cohort. The American Journal of Clinical Nutrition, 97(3), 517-523. doi:10.3945/ajcn.112.050997

Flores A, González-Celis R., \& Valencia O. (2010) Validation of the SelfEfficacy scale for healthy behavior in healthy Mexican children. Psicología y Salud, 20(1), 23-30.

Forshee, R., Anderson, P., \& Storey, M. (2008) Sugar-sweetened beverages and body mass index in children and adolescents: a meta-analysis. The American Journal of Clinical Nutrition, 87(1), 1662-1671. doi:10.1093/ajen/87.6.1662

Fowler, S., Williams, K., Resendez, R., Hunt, K., Hazuda, H., \& Stern, M. (2008). Fueling the obesity epidemic? Artificially sweetened beverage use and long-term weight gain. Obesity, 16(8), 1894-1900. doi:10.1038/oby.2008.284

Franco, K., \& Miramontes, E. (2013). Frecuencia de consumo de alimentos, índice de masa corporal y porcentaje de grasa en estudiantes universitarios: un estudio longitudinal. CienciaUat, 7(2), 18-22.

Frank, G., Oberndorfer, T., Simmons, A., Paulus, M., Fudge, J., Yang, T., \& Kaye, W. (2008). Sucrose activates human taste pathways differently from artificial sweetener. Neuroimage, 39(4), 1559-1569. doi:10.1016/j.neuroimage.2007.10.061 
García, C., López, A., Moreno, A., Miranda, C., \& Zepeda, A. (2018). Estrategias para la disminución del consumo de bebidas endulzadas. Revista Española de Nutrición Humana y Dietética, 22(2), 169-179. doi:10.14306/renhyd.22.2.426

Guzmán, S., \& Gómez, P. (2011). Discriminant Analysis of the Self-efficacy Inventory for Weight Control in Mexican Adolescents. Psicología y Salud, 21(2), 157-164. doi:10.25009/pys.v21i2.568

Hughes, S., Power, T., \& O'Connor, T. (2015). Executive functioning, emotion regulation, eating self-regulation, and weight status in lowincome preschool children: how do they relate? Appetite, 89, 1-9. doi:10.1016/j.appet.2015.01.009

Johnson, F., Pratt, M., \& Wardle, J. (2012). Dietary restraint and selfregulation in eating behavior. International Journal of Obesity, 36(5), 665 - 674. doi:10.1038/ijo.2011.156

Lugli, Z., Arzolar, M., \& Vivas, E. (2009). Construcción y validación del Inventario de Autorregulación del Peso: validación preliminar. Psicología y 281-287. doi:10.25009/pys.v19i2.638

Malik, V., Popkin, B., Bray, G., Després, J., Willett, W., \& Hu, F. (2010) SugarSweetened Beverages and Risk of Metabolic Syndrome and Type 2 Diabetes. Diabetes Care, 33(11), 2477-2483. doi:10.2337/dc10-1079

Mattes, R., \& Popkin, B. (2008). Nonnutritive sweetener consumption in humans: effects on appetite and food intake and their putative mechanisms. The American Journal of Clinical Nutrition, 89(1), 1-14. doi:10.3945/ajen.2008.26792

Montero, I., \& León, O. (2002). Clasificación y descripción de las metodologías de investigación en psicología. Revista Internacional de Psicología Clínica y de la Salud, 2(3), 503-508.

Organización Mundial de la Salud (OMS). (2018). Obesidad y sobrepeso. Recuperado el 12 de agosto 2018, de http://www.who.int/es/newsroom/fact-sheets/detail/obesity-and-overweight

Palacios, J. (2015). Estimación psicométrica de la escala de autoeficacia ante conductas de riesgo para adolescentes en México. Psychosocial Intervention, 1, 1-7. doi:10.1016/j.psi.2014.11.004

Palacios, J. (2019a). Predictors of personality and self-efficacy of sexual risk behavior in Mexican adolescents. Annals of Psychology, 35(1), 131 139. doi:10.6018/analesps.35.1.319471

Palacios, J. (2019b). Desarrollo y validación de una escala de evaluación de la autoregulación del consumo de bebidas endulzadas. Health and Addictions/Saludy Drogas, 19(1), 99-109. doi:10.21134/haaj.v19i1.419

Palacios, J., \& Bustos, A. J. M. (2012). La teoría como promotor para el desarrollo de intervenciones psicoambientales. Psychosocial Intervention, 21(3), 245- 257. doi:/10.5093/in2012a22

Palacios, J., Ramírez, V., Anaya, A., Hernández, H., \& Martínez, M. (2017). Evaluación psicométrica de la escala de autoeficacia del consumo alimenticio. Revista Chilena de Nutrición, 44(1), 95-102. doi:10.4067/S0717-75182017000100013

Palacios J., \& Ramírez V. (2016). Estudio comparativo de la autoeficacia saludable en las conductas alimenticias de riesgo. Revista Psicología Iberoamericana, 24(2), 17-25.
Palacios, J., \& Soler, A. L. (2017). Preliminary study of the comparative profile of financial control in university young people. Ciencias Psicológicas, 11(2), 201- 212. doi:10.22235/cp.v11i2.1491

Raffaelli, M., Crockett, L., \& Shen, Y. (2005). Developmental stability and change in self-regulation from childhood to adolescence. The Journal of Genetic Psychology, 166(1), 54-76. doi:10.3200/GNTP.166.1.54-76

Rodríguez, M., Avalos, M., \& López, C. (2014). Consumo de bebidas de alto contenido calórico en México: un reto para la salud pública. Salud en Tabasco, 20(1), 28-33.

Román, Y., Díaz, B., Cárdenas, M., \& Lugli, Z. (2007). Construction and validation of the perceived self-efficacy inventory for weight control. Clinica y Salud, 18(1), 45-56.

Ruíz, V., Berrocal, C., López, A., \& Rivas, T. (2003). Self-efficacy in the control behavior intake. Adaptation to Spanish of the Eating Self-Efficacy Scale. Psicothema, 15(1), 36-40.

Secretaría de Salud. (2011). Reglamento de la Ley General de Salud en Materia de Investigación para la Salud. Recuperado Febrero 15, 2017,

de

www.salud.gob.mx/unidades/cdi/nom/compi/rlgsmis.html

Secretaría de Salud, Instituto Nacional de Salud Pública (INSP). (2016) Encuesta Nacional de Salud y Nutrición de Medio Camino 2016 (ENSANUT). Informe final. Ciudad de México. [citado el 02 de abril 2019]. Disponible en: https://www.gob.mx/cms/uploads/attachment/file/209093/ENSAN $\underline{\text { UT.pdf }}$

Uscanga, Y., González, T., Del Moral, L., \& Hernández, N. (2017). Obesidad y autorregulación de la actividad física y la alimentación en estudiantes universitarios: un estudio longitudinal. MHSalud: Movimiento Humano y Salud, 14(1), 1-16.

Wardle, J., Guthrie, C. A., Sanderson, S., \& Rapoport, L. (2001). Development of the children's eating behaviour questionnaire. The Journal of Child Psychology and Psychiatry and Allied Disciplines, 42(7), 963-970.

Wilson-Barlow L., Hollins T., \& Clopton J. (2014). Construction and validation of the healthy eating and weight self-efficacy (HEWSE) scale. Eating Behavior, 15 (13), 490-492. doi:10.1016/j.eatbeh.2014.06.004 